\title{
India will Reach Moon, will Orthopaedics Reach that Height?
}

\section{Dilip D Shah*}

\section{Department of Orthopaedics, University of Bombay, India}

*Corresponding Author: Dilip D Shah, Department of Orthopaedics, University of Bombay, India.

Received: July 25, 2019; Published: September 01, 2019

DOI: 10.31080/ASOR.2019.02.0096

It has been quite a historical event that India makes headway to the moon, similarly Orthopaedics has reached to that soaring height and is progressing by leaps and bounds.

\section{Traumatology}

Anatomy specific implants are in vogue with specificity and low profile implants and also Locked Compression Plating along with variable angles make the life of the surgeon quite easy. Alas in the race of newer implants the young surgeons are using the fancy implants without employing the principles and using the discrimination of choosing the correct implant. Hence there are implant failures. Robotic surgery is making headway but there is a big learning curve and cost benefit ratio is to be observed. Lowintensity pulsed ultrasound healing in fastidious and delayed and non - union of fractures is nowadays not preferred for the cost benefit ratio and inability to prove its efficacy. Biodegradable implants especially for Intra-articular fractures are being studied and will find a definite place in limited indications.

Arthroplasty: Robotic surgery and Computer assisted surgeries are giving specific outcomes to the surgery thereby giving perfect results. Newer dimensions are for moderate arthritis involving mainly medial joint compartment where uni compartmental replacements are gaining popularity where a newer surgery of enhancing the shock absorber effect. The Calypso is designed to provide support outside of the knee joint without altering the anatomy or removing any tissue from the knee itself.

In cartilage replacement surgery MACI is newer method compared to old generations of ACI such as Carticel, which had been on the market for two decades. MACI received FDA approval for it in December 2016 and was launched it in early 2017. Cultured cartilage cell membrane if trans plated in the areas of worn-out or cartilage depleted areas in two stages.

\section{Spinal surgery}

"Never before in history has innovation offered promise of so much to so many in so short a time".
Bill Gates' wise words ring true for the innovative technologies being developed in spine surgery. So far in metallurgy for spinal surgery implants has been Cobalt chromium, Titanium or Stainless Steel.

There is influx of new alloys, such as molybdenum-rhenium that provides strong and more durable constructs, while allowing for smaller sized implants.

3 D printed implants are again a newer technology so is the Endoscopic spinal surgery which is slowly replacing traditional open and also Micro surgery.

All the newer technologies with steep learning curve \& cost benefit ratio will meet the new challenges the patient's outcome will definitely improve, whether or not we reach to moon but the moon is in near vicinity.

\section{Volume 2 Issue 10 October 2019 (C) All rights are reserved by Dilip D Shah.}

